\title{
Engineering the $\mathrm{MoSe}_{2} / \mathrm{WS}_{2}$ hybrids to replace the
}

\section{scarce platinum electrode for hydrogen-evolution}

\section{reactions and dye-sensitized solar cells}

Dhanasekaran Vikramana, Sajjad Hussain ${ }^{b, c}$, Supriya A. Patilc, Linh Truongd, Alvira

Ayoub Arbabe, Sung Hoon Jeong ${ }^{e}$, Seung-Hyun Chun ${ }^{d}$, Jongwan Jung ${ }^{b, c}$, Hyun-Seok $\mathrm{Kim}^{a^{*}}$

a. Division of Electronics and Electrical Engineering, Dongguk University-Seoul, Seoul 04620, Republic of Korea.

b. Hybrid Materials Center (HMC), Sejong University, Seoul 05006, Republic of Korea.

c. Department of Nanotechnology and Advanced Materials Engineering, Sejong University, Seoul 05006, Republic of Korea.

d. Department of Physics, Sejong University, Seoul 05006, Republic of Korea.

e. Department of Organic and Nano Engineering, Hanyang University, Seoul 04763, Republic of Korea.

*Email: hyunseokk@dongguk.edu; Tel: +82-2-2260-3996; Fax: +82-2-2277-8735 
*Email: hyunseokk@dongguk.edu; Tel: +82-2-2260-3996; Fax: +82-2-2277-8735

Number of pages: 27

Number of figures: 13

Number of tables: 5

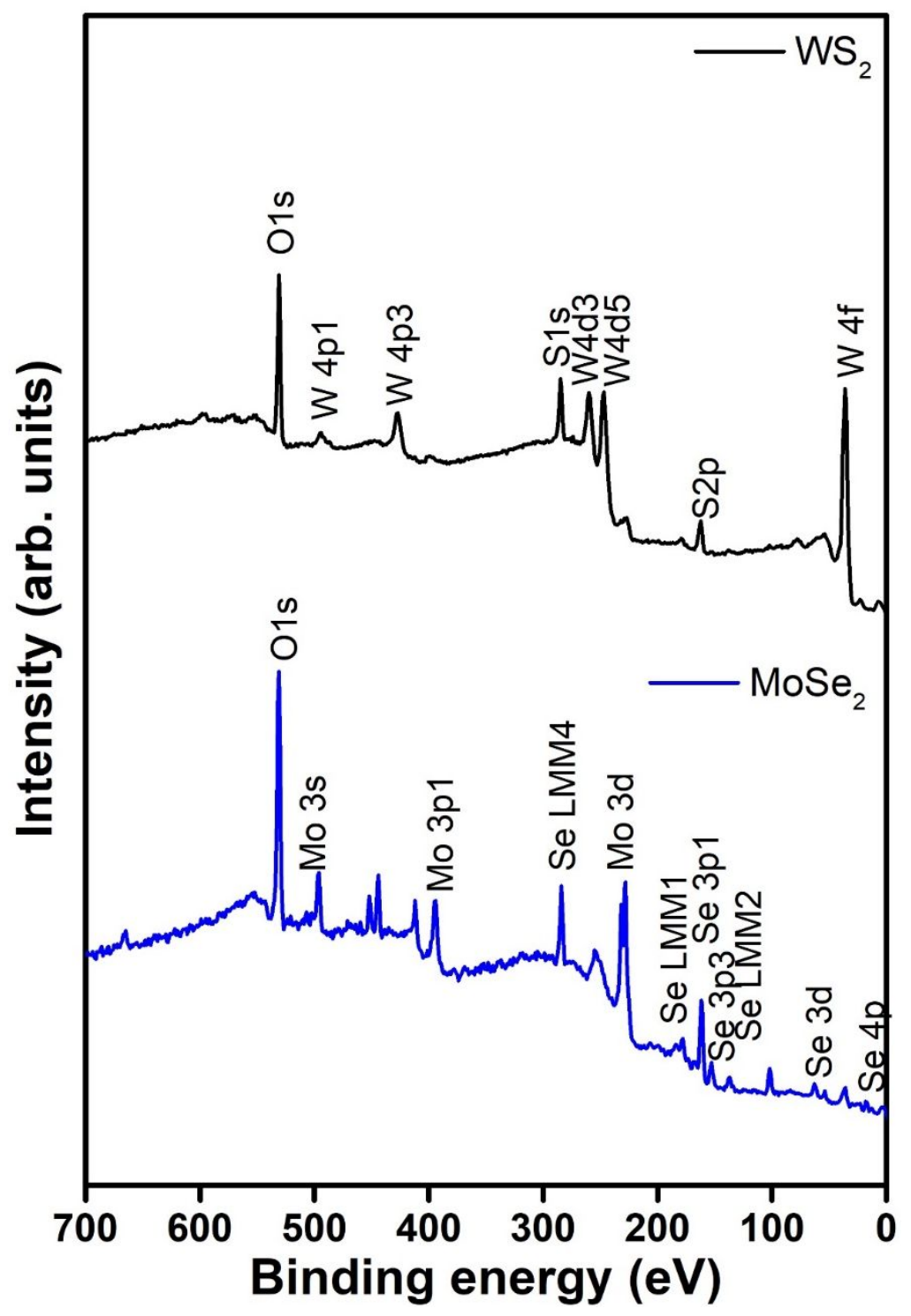

Figure S1. XPS survey spectra for $\mathrm{MoSe}_{2}$ and $\mathrm{WS}_{2}$ structures. 


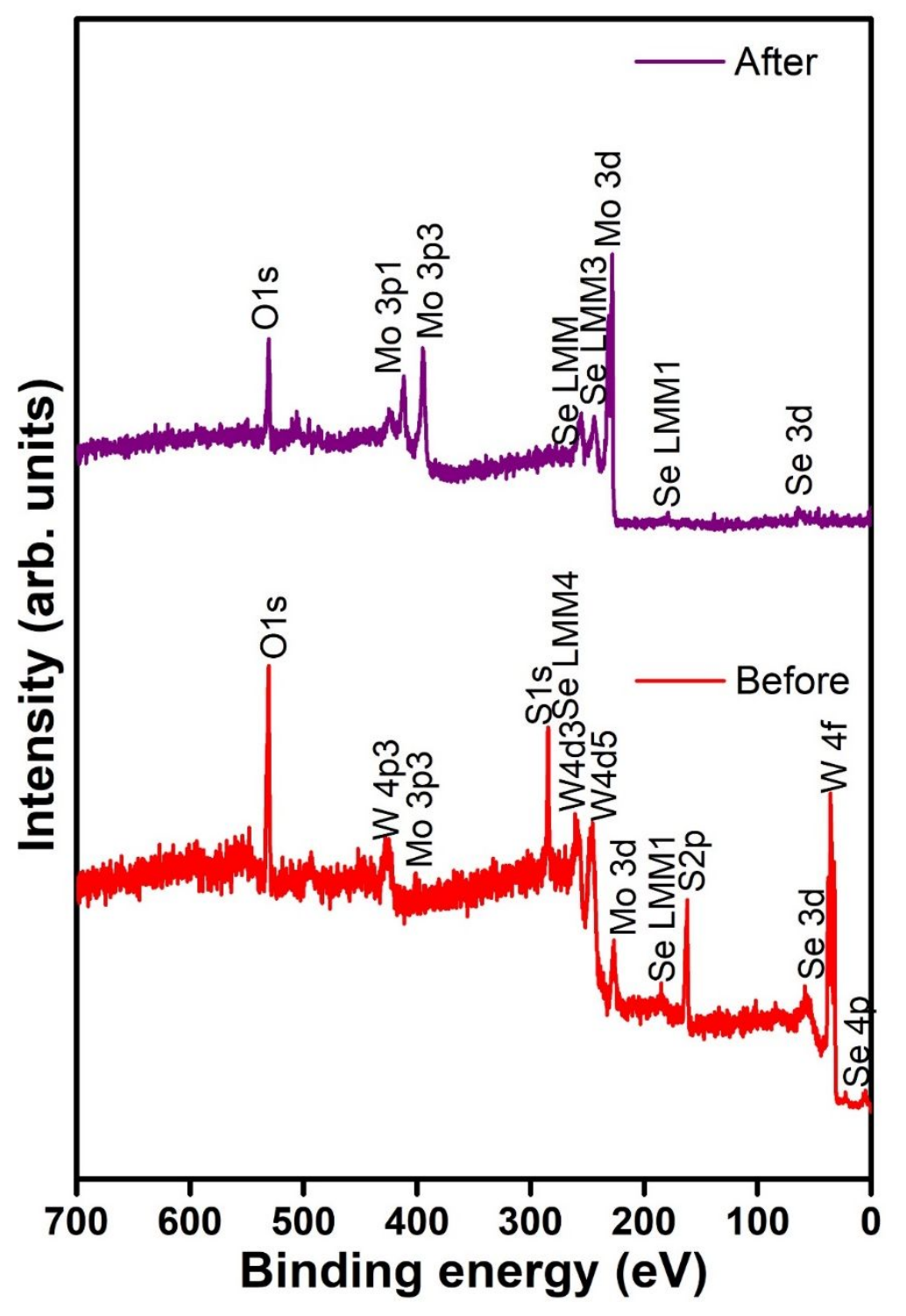

Figure S2. XPS survey spectra of before and after etching process for $\mathrm{MoSe}_{2} / \mathrm{WS}_{2}$ hybrid. 


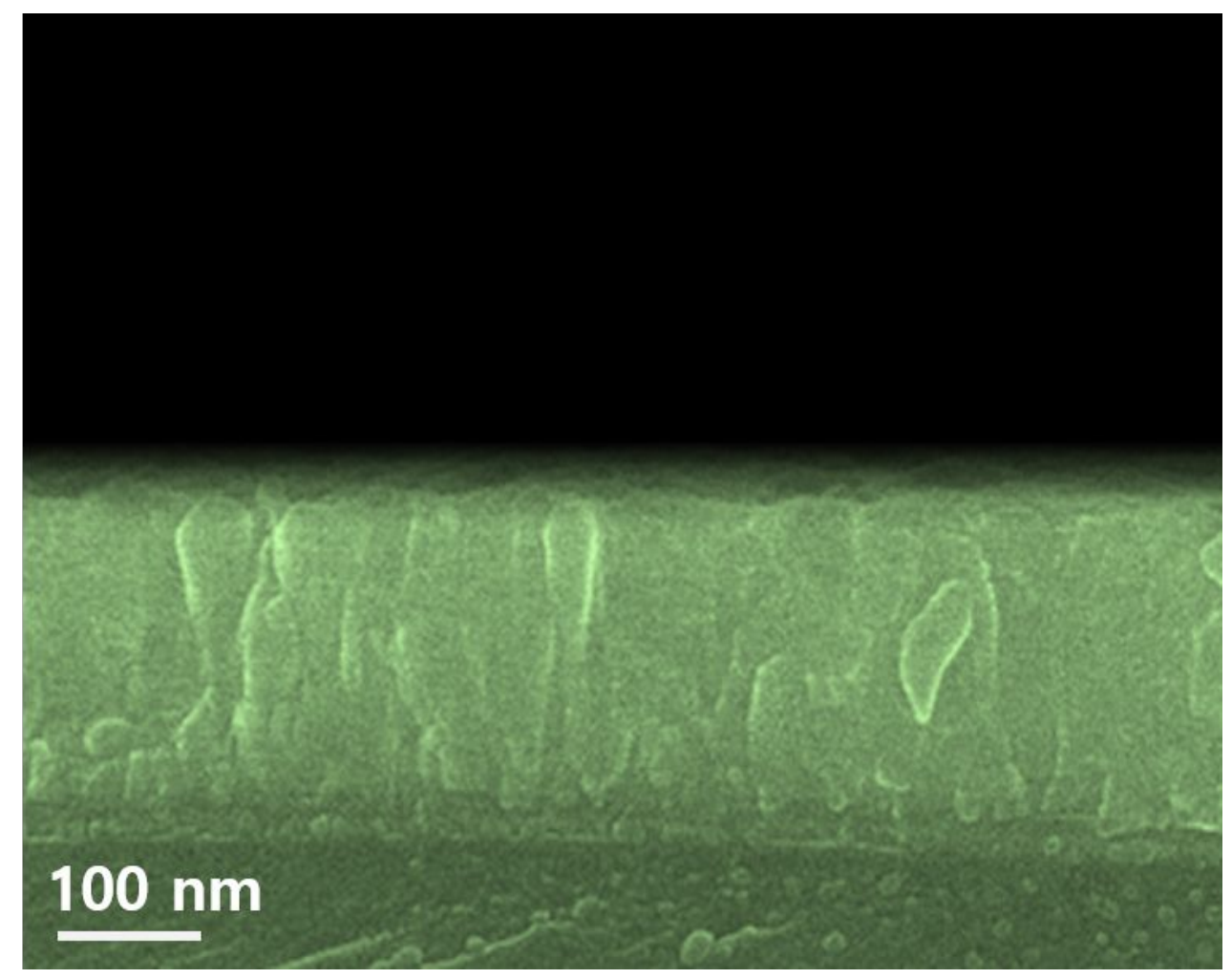

Figure S3. Cross section FE-SEM image for $\mathrm{H} 1 \mathrm{MoSe}_{2} / \mathrm{WS}_{2}$ hybrid. 

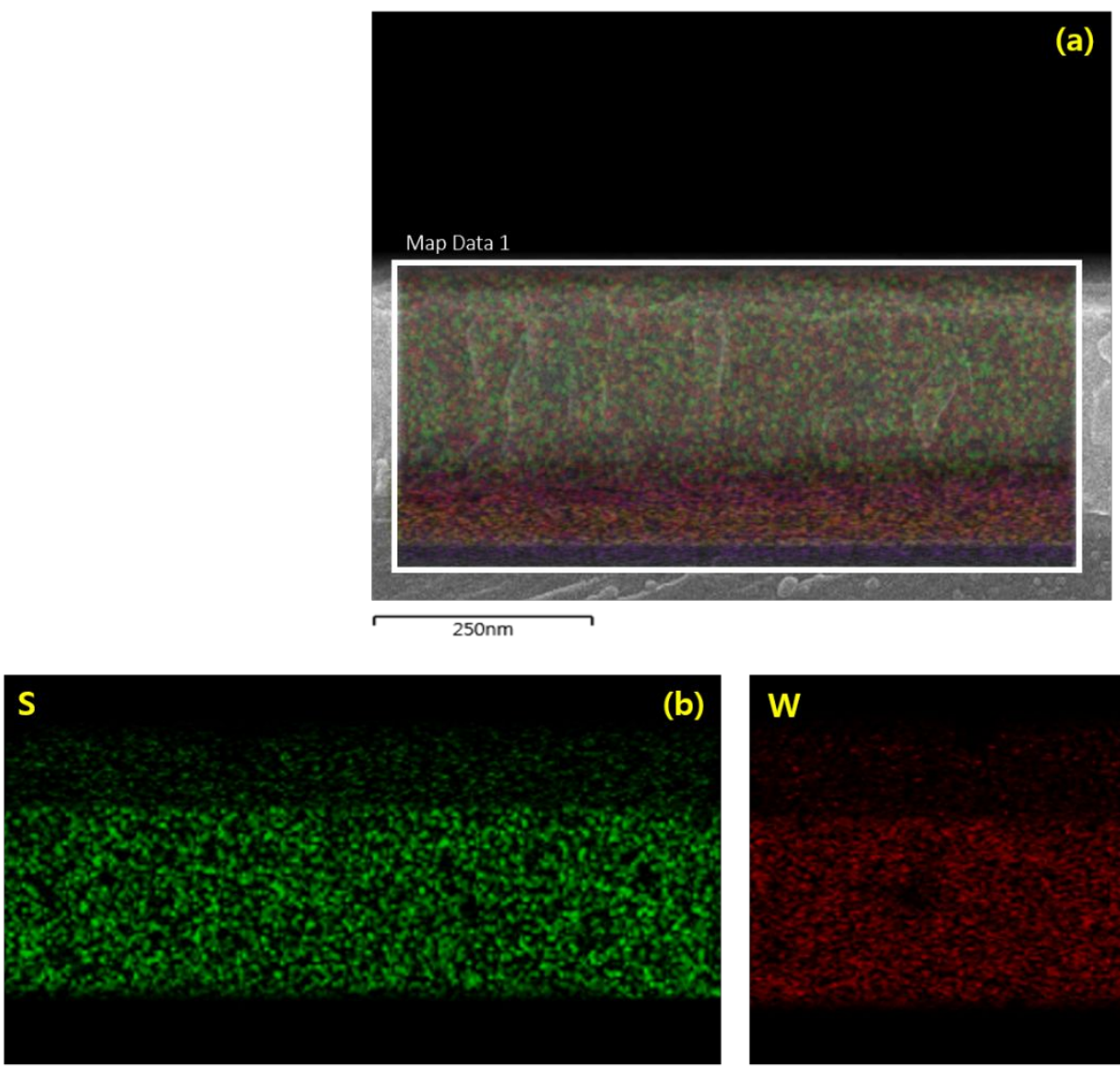

(d)

Se

(e)

Figure S4. (a) Elemental mapping image and their (b) S, (c) W, (d) Mo and (e) Se elements distributions for $\mathrm{H} 1 \mathrm{MoSe}_{2} / \mathrm{WS}_{2}$ hybrid. 

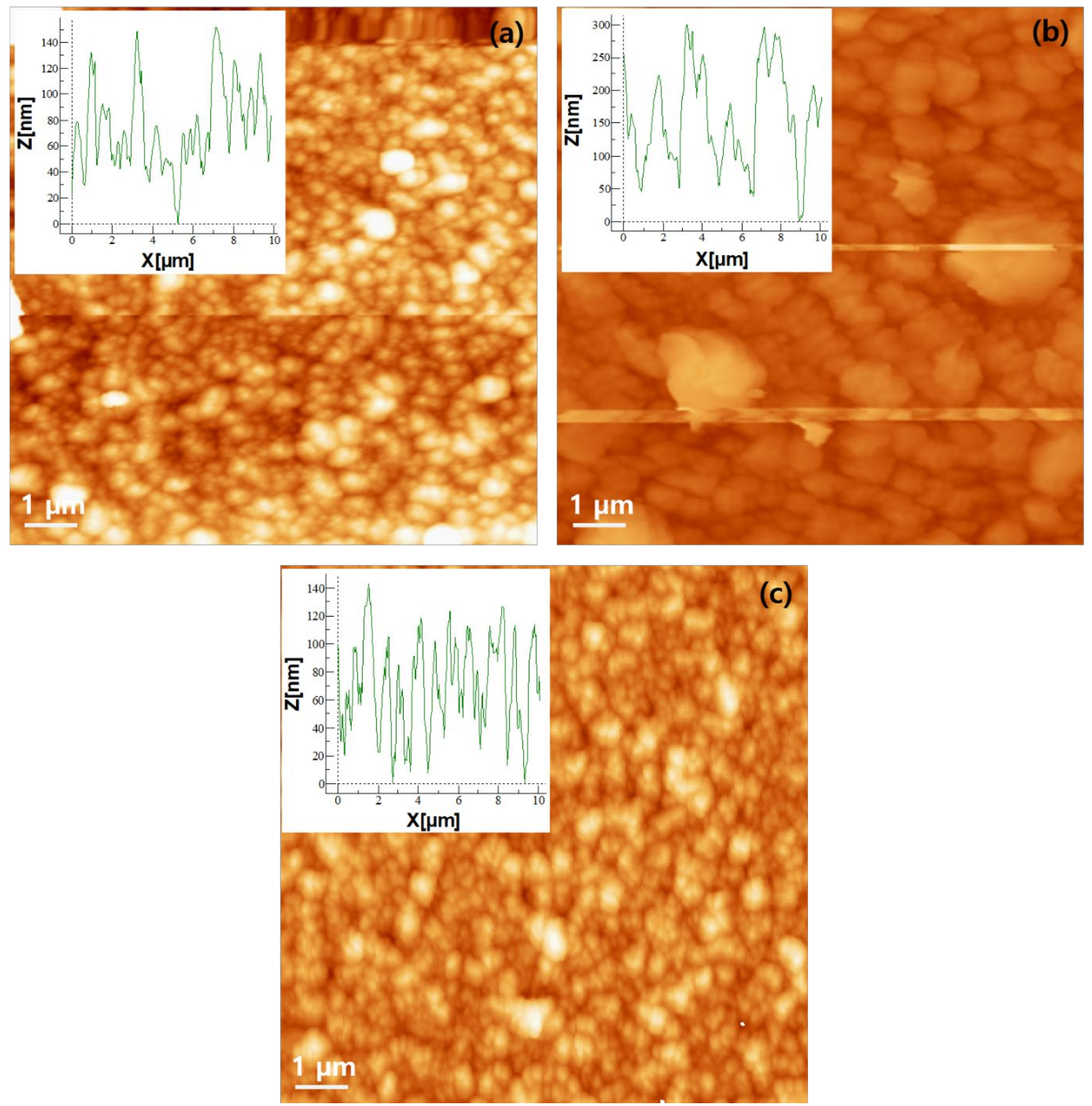

Figure S5. AFM images of (a) $\mathrm{MoSe}_{2}$, (b) $\mathrm{WS}_{2}$ and (c) the $\mathrm{MoSe}_{2} / \mathrm{WS}_{2}$ hybrid with the inset of corresponding surface profile. 


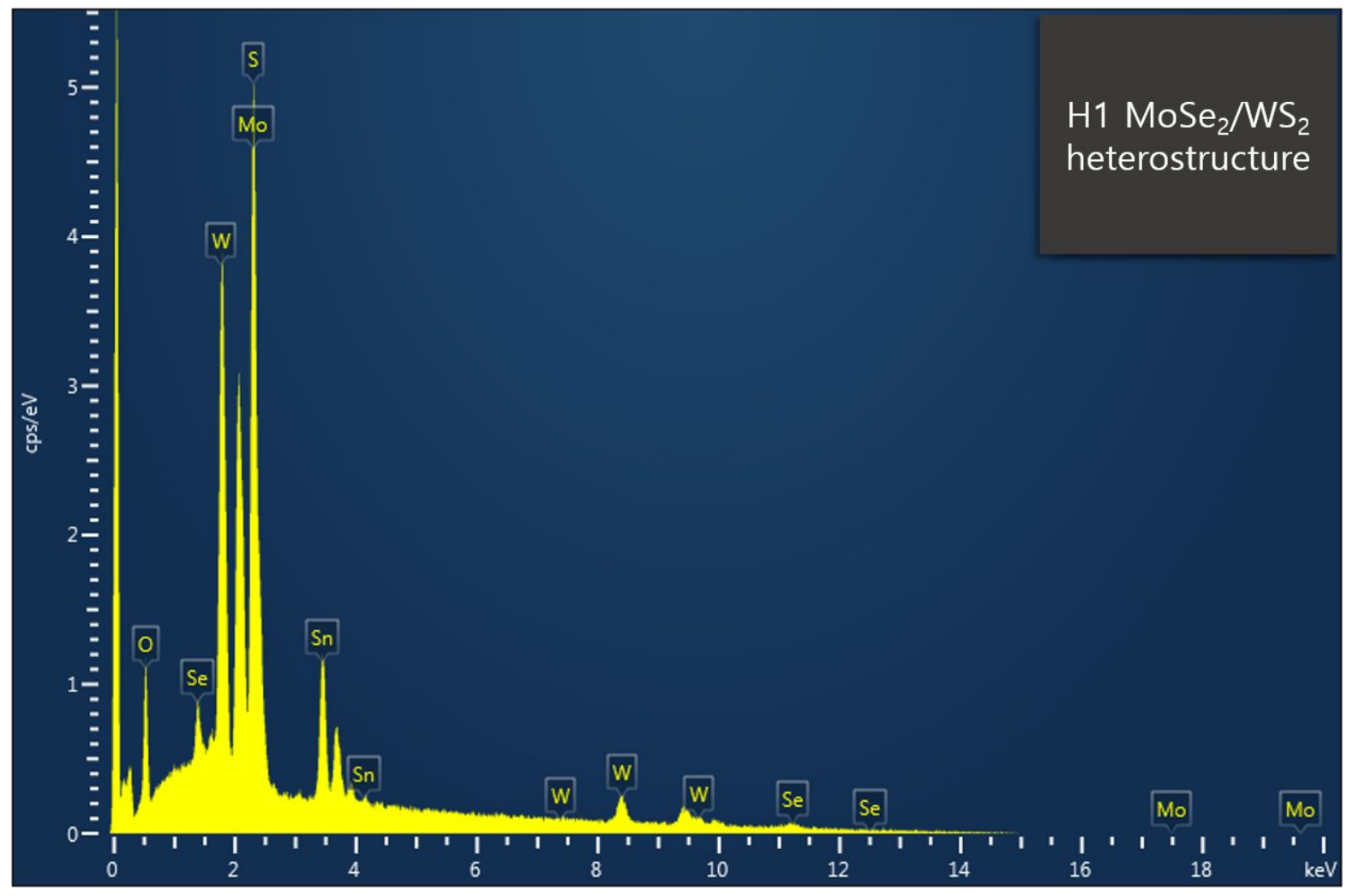

Figure S6. Energy dispersive elemental analysis profile for $\mathrm{H} 1 \mathrm{MoSe}_{2} / \mathrm{WS}_{2}$ hybrid. 


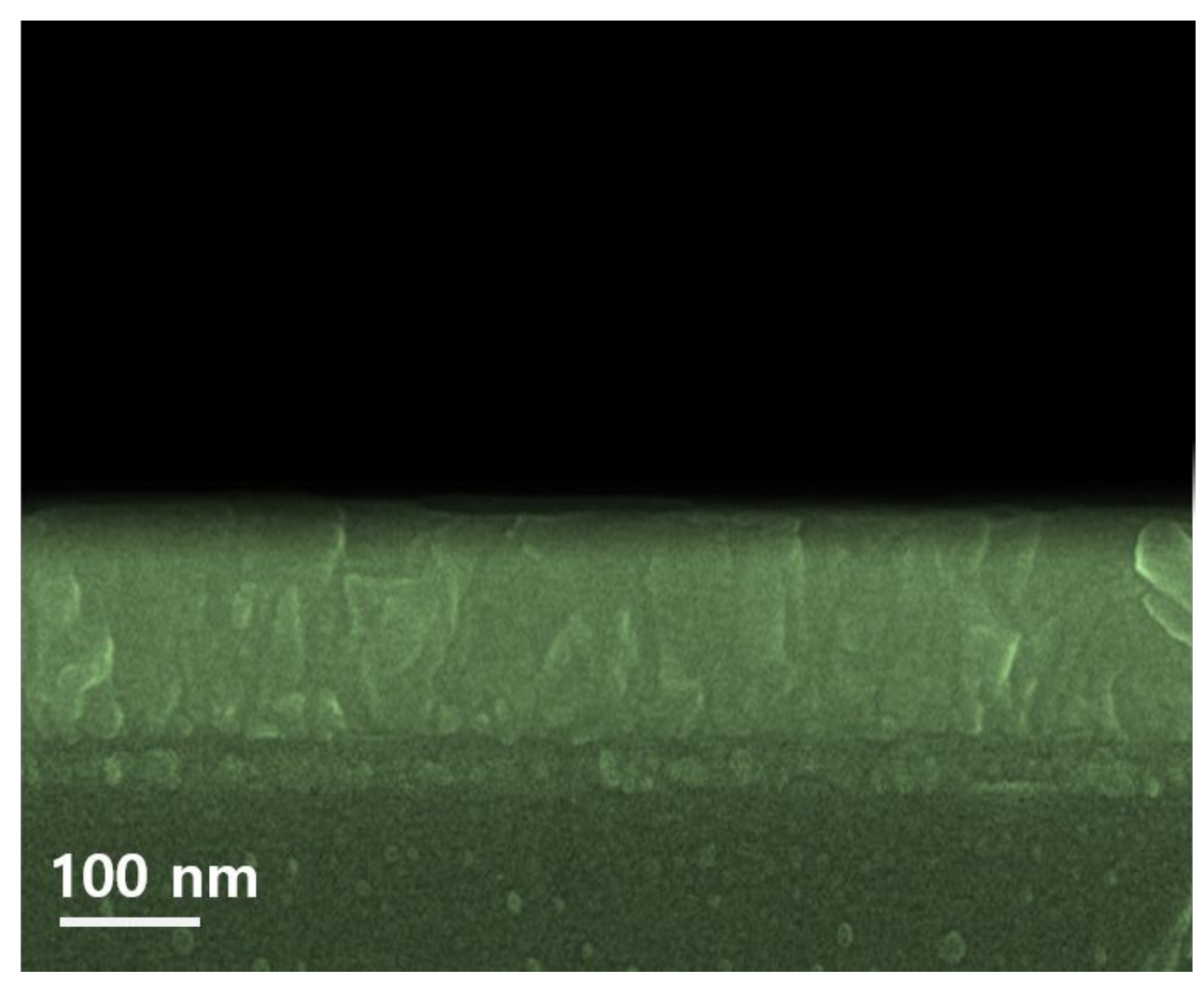

Figure S7. Cross section FE-SEM image for $\mathrm{H} 2 \mathrm{MoSe}_{2} / \mathrm{WS}_{2}$ hybrid. 


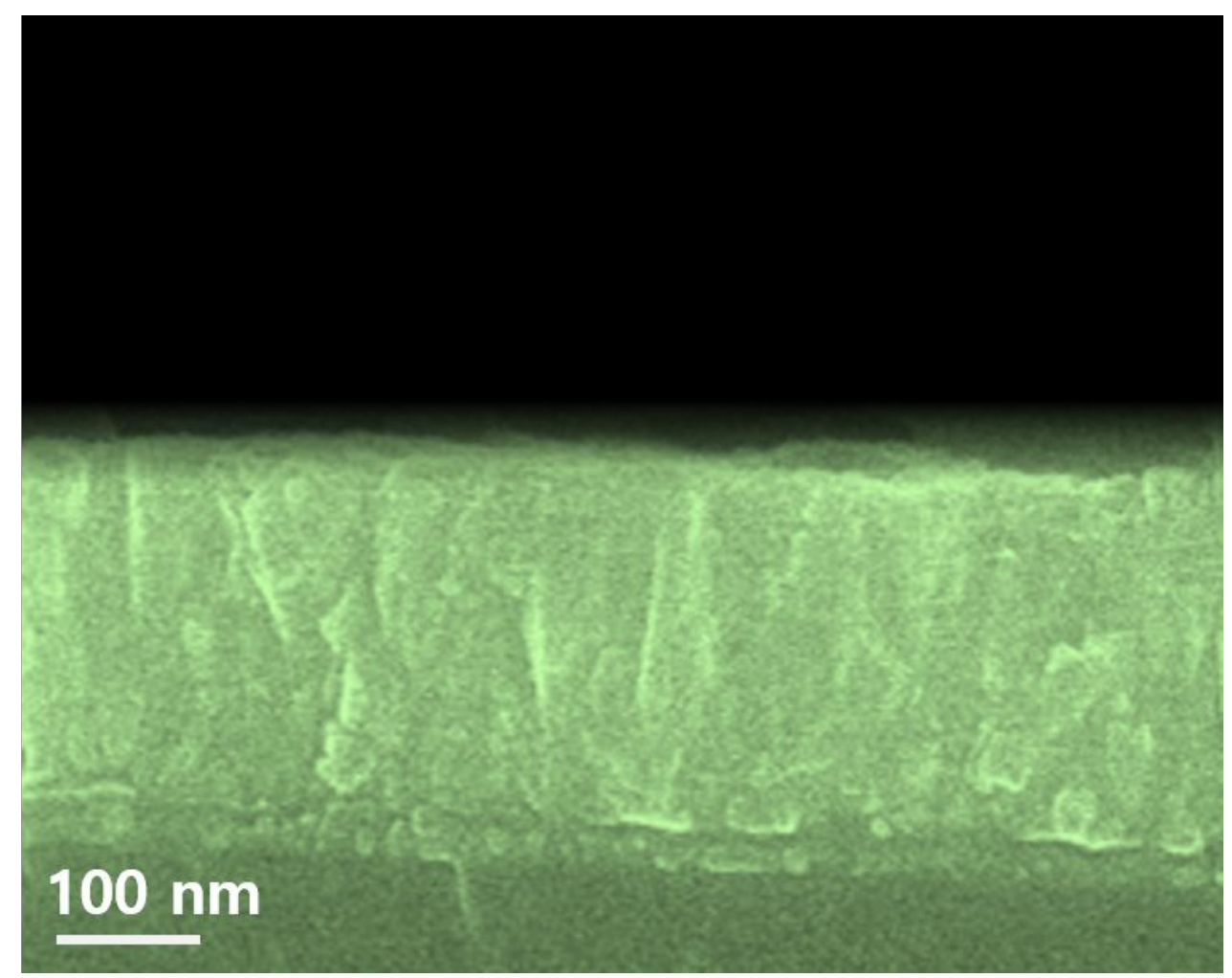

Figure S8. Cross section FE-SEM image for $\mathrm{H} 3 \mathrm{MoSe}_{2} / \mathrm{WS}_{2}$ hybrid. 


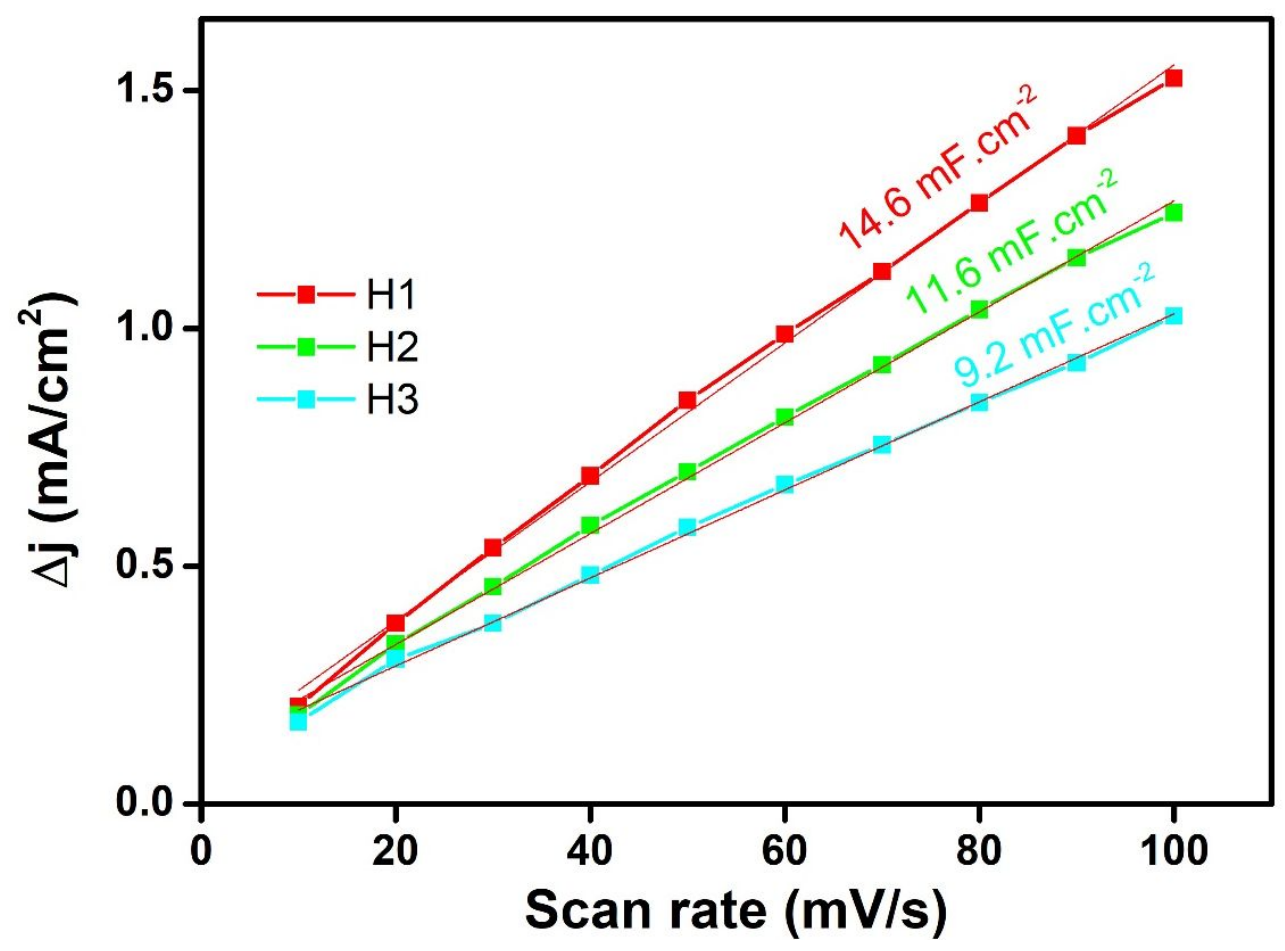

Figure S9. Linear relation between scan rate versus current density difference $\left(\Delta j=j_{c}-j_{a}\right)$ at $0.125 \mathrm{~V}$ vs RHE for $\mathrm{H} 1, \mathrm{H} 2$ and $\mathrm{H} 3 \mathrm{MoSe}_{2} / \mathrm{WS}_{2}$ hybrid.

\section{ECSA calculation:}

ECSA were estimated using the following relation:

$E C S A=C_{d l} / C_{S}$

where, $\mathrm{C}_{\mathrm{dl}}$ is the double layer capacitance and $\mathrm{C}_{\mathrm{s}}$ is the specific capacitance which is equal to $0.035 \mathrm{mF} . \mathrm{cm}^{-2}$ for $0.5 \mathrm{M} \mathrm{H}_{2} \mathrm{SO}_{4} \cdot{ }^{1} \mathrm{C}_{\mathrm{dl}}$ values were estimated by the relation $i_{d}=v \mathrm{C}_{\mathrm{dl}}$, where $i_{d}$ is the current differences from double layer anodic and cathodic current density, $v$ is the scan rate and $\mathrm{C}_{\mathrm{dl}}$ value evaluated from the fitted slope value. 


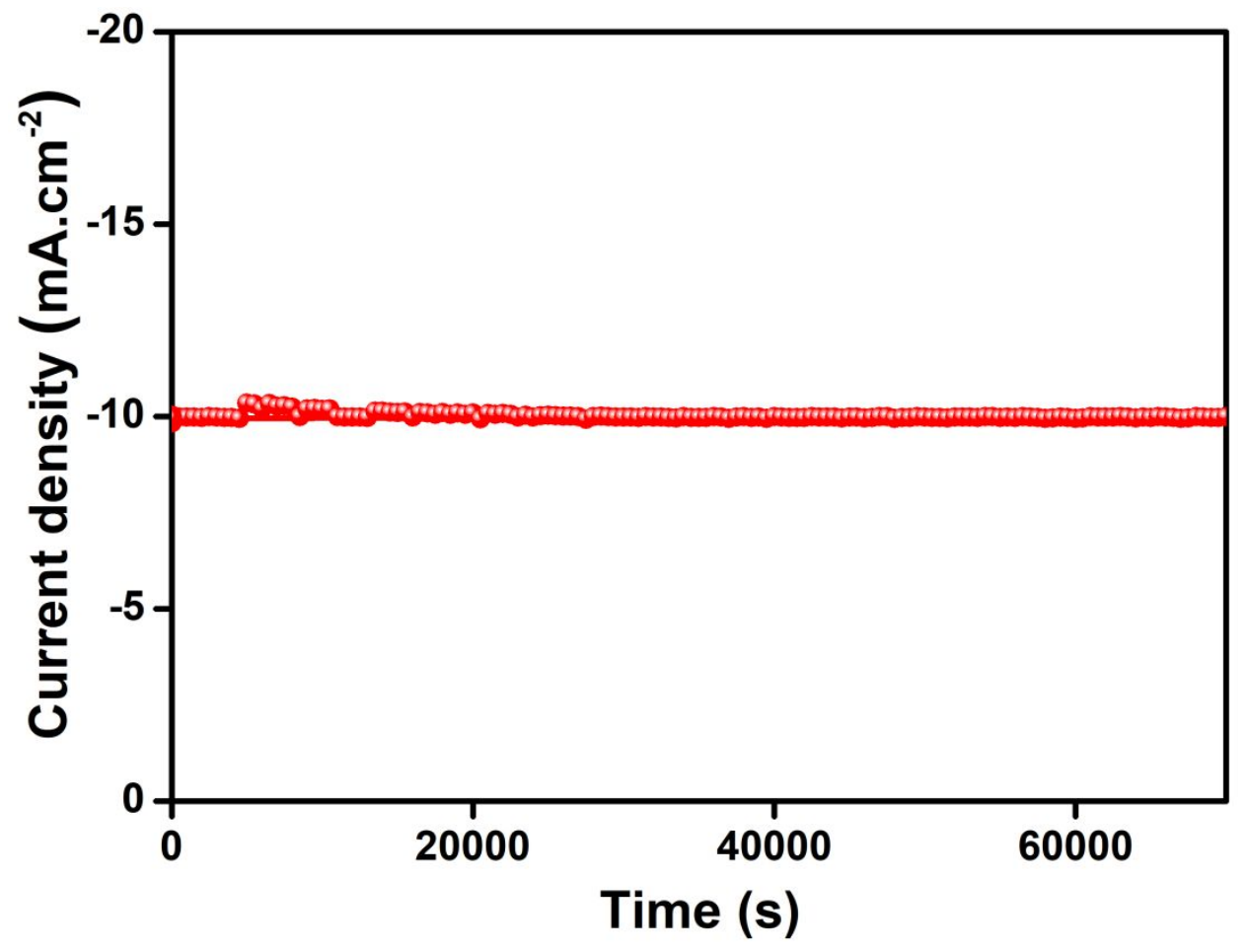

Figure S10. Stability chronoamperometric profile using the constant overpotential (75 $\mathrm{mV}$ ) for $\mathrm{H} 1 \mathrm{MoSe}_{2} / \mathrm{WS}_{2}$ hybrid. 


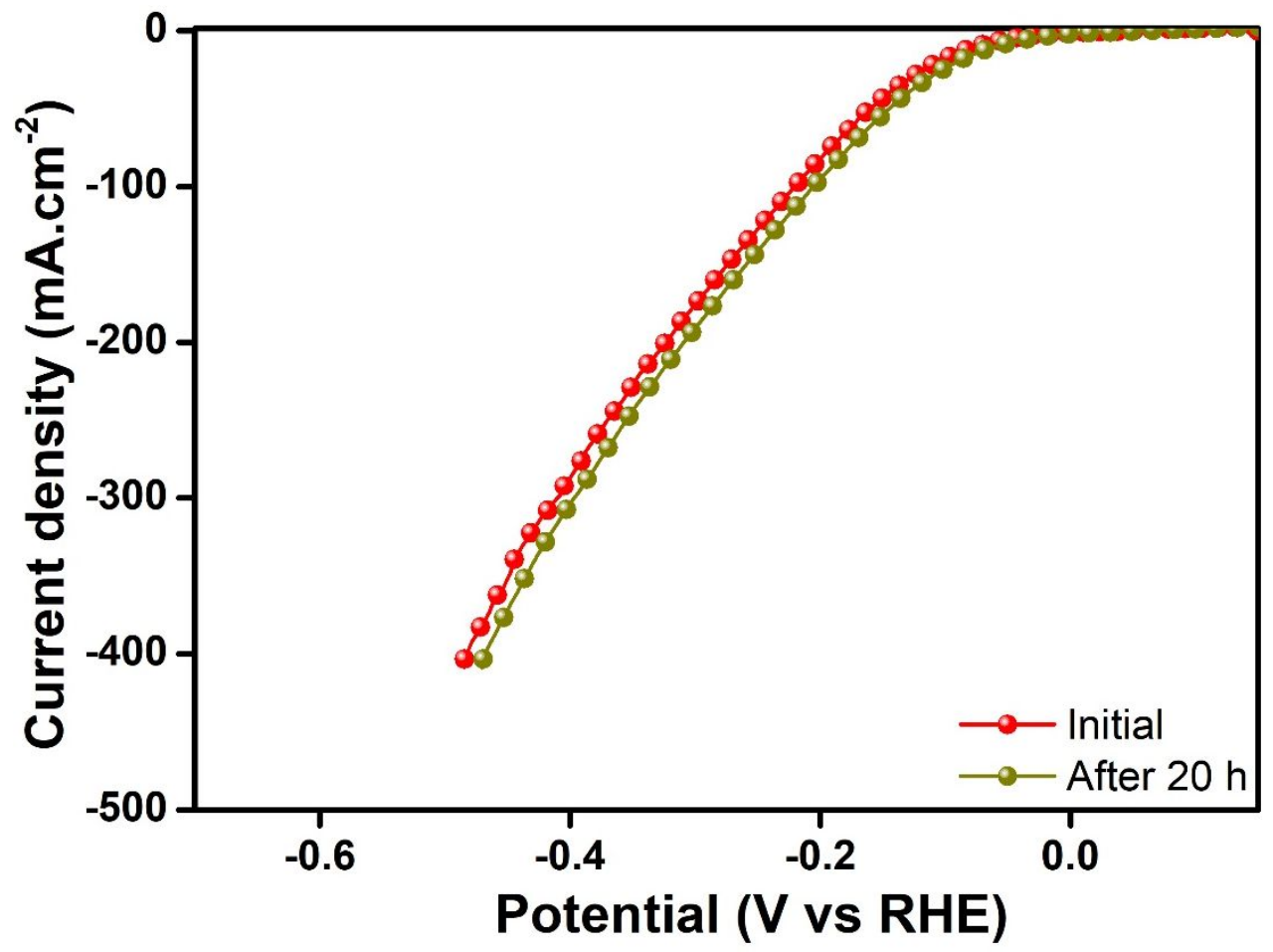

Figure S11. Polarization curve for before and after $20 \mathrm{~h}$ continuous hydrogen evolution process for $\mathrm{H} 1 \mathrm{MoSe}_{2} / \mathrm{WS}_{2}$ hybrid. 


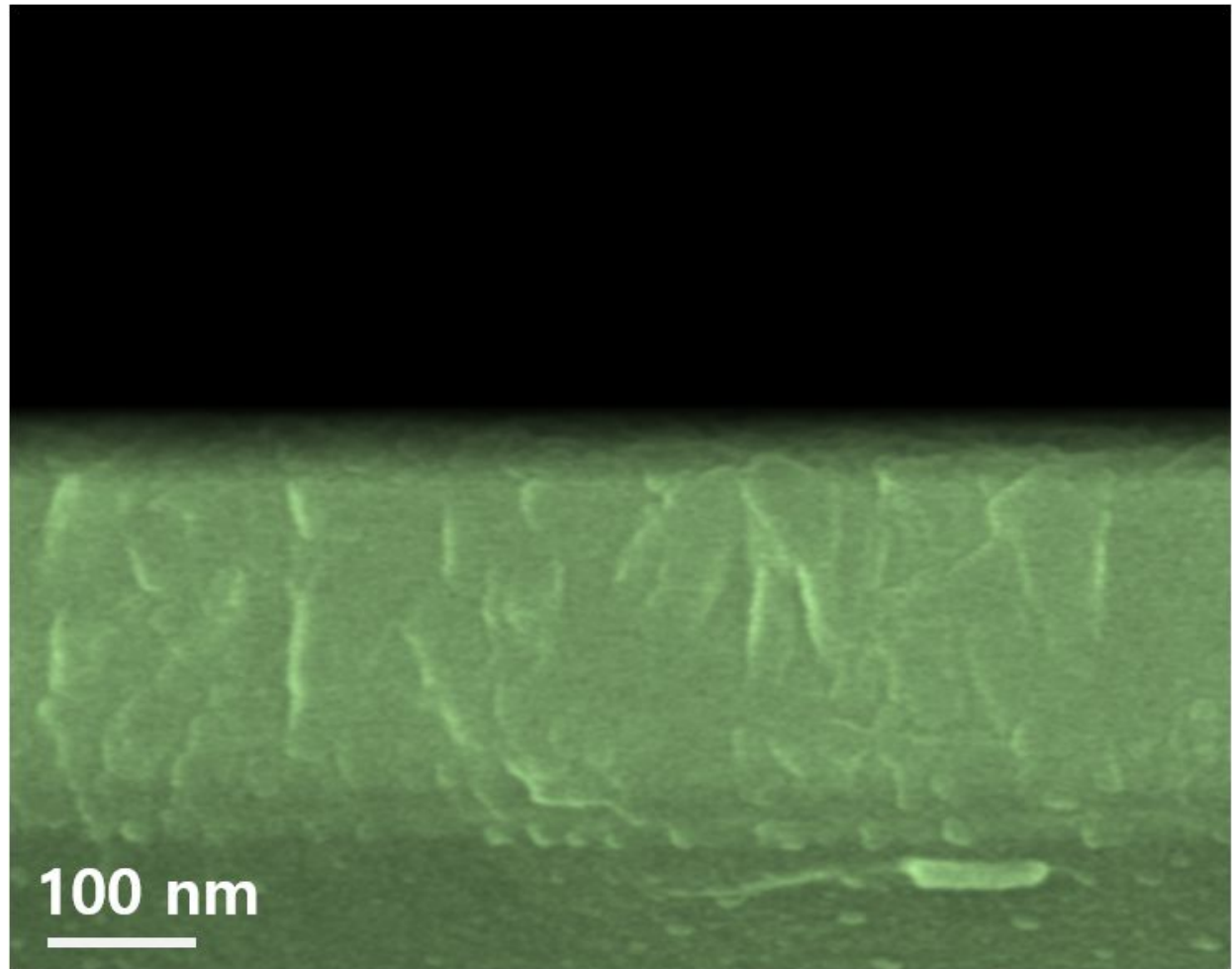

Figure S12. Cross section FE-SEM image after $20 \mathrm{~h}$ continuous hydrogen evolution process for $\mathrm{H} 1 \mathrm{MoSe}_{2} / \mathrm{WS}_{2}$ hybrid. 


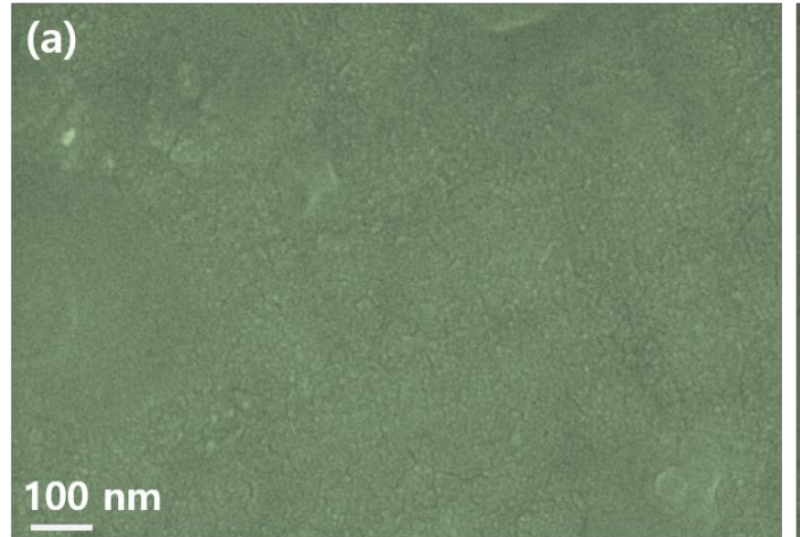

(b)

Figure S13(a-b). FE-SEM images of $\mathrm{H} 1 \mathrm{MoSe}_{2} / \mathrm{WS}_{2}$ hybrid after $20 \mathrm{~h}$ continuous hydrogen evolution process. 
Table S1. The characteristic DSSC parameters of various counter electrodes

\begin{tabular}{c|cccc}
\hline \multirow{2}{*}{ Electrode } & $\begin{array}{c}\boldsymbol{P C E} \\
(\%)\end{array}$ & $\begin{array}{c}\boldsymbol{F F} \\
(\%)\end{array}$ & $\begin{array}{c}\boldsymbol{V}_{\boldsymbol{O C}} \\
(\boldsymbol{V})\end{array}$ & $\begin{array}{c}\boldsymbol{J}_{\boldsymbol{S C}} \\
\left(\boldsymbol{m} \boldsymbol{A} \cdot \mathbf{c m}^{-2}\right)\end{array}$ \\
\hline MoSe $_{2}$ & 7.42 & 67.39 & 0.64 & 17.30 \\
$W S_{2}$ & 6.73 & 67.13 & 0.66 & 15.23 \\
$H 1$ & 9.92 & 65.15 & 0.66 & 23.10 \\
$H 2$ & 8.23 & 62.69 & 0.62 & 21.28 \\
$H 3$ & 7.63 & 66.86 & 0.65 & 17.58 \\
$P t$ & 8.73 & 68.9 & 0.71 & 17.84 \\
\hline
\end{tabular}

$\mathrm{PCE}=$ power conversion efficiency; $\mathrm{FF}=$ fill factor; $V_{O C}=$ open circuit photovoltage; $J_{S C}=$ short circuit photocurrent density. 
Table S2. Stability outputs for DSSC using various electrodes

\begin{tabular}{|c|c|c|c|c|c|}
\hline Electrode & Experiment & $\begin{array}{c}P C E \\
(\%)\end{array}$ & $\begin{array}{l}F F \\
(\%)\end{array}$ & $\begin{array}{l}V_{O C} \\
(V)\end{array}$ & $\begin{array}{c}J_{S C} \\
\left(m A \cdot c m^{-2}\right)\end{array}$ \\
\hline \multirow{3}{*}{$\mathrm{MoSe}_{2}$} & I & 7.421 & 67.394 & 0.636 & 17.308 \\
\hline & II & 7.423 & 67.374 & 0.636 & 17.311 \\
\hline & III & 7.428 & 67.481 & 0.636 & 17.309 \\
\hline \multirow{3}{*}{$W S_{2}$} & $\mathrm{I}$ & 6.734 & 67.133 & 0.658 & 15.234 \\
\hline & II & 6.729 & 67.161 & 0.658 & 15.227 \\
\hline & III & 6.738 & 67.223 & 0.658 & 15.233 \\
\hline \multirow{3}{*}{$H 1$} & I & 9.922 & 65.156 & 0.659 & 23.101 \\
\hline & II & 9.923 & 65.098 & 0.659 & 23.132 \\
\hline & III & 9.927 & 65.089 & 0.659 & 23.145 \\
\hline \multirow{3}{*}{$H 2$} & I & 8.237 & 62.695 & 0.617 & 21.280 \\
\hline & II & 8.239 & 62.757 & 0.617 & 21.279 \\
\hline & III & 8.241 & 62.765 & 0.618 & 21.248 \\
\hline \multirow{3}{*}{$H 3$} & I & 7.633 & 66.864 & 0.649 & 17.585 \\
\hline & II & 7.633 & 66.817 & 0.650 & 17.576 \\
\hline & III & 7.627 & 66.972 & 0.649 & 17.548 \\
\hline
\end{tabular}


Table S3. DSSC performances of various 2D counter electrodes

\begin{tabular}{|c|c|c|c|c|c|}
\hline Type of counter electrodes & $\begin{array}{r}\text { PCE } \\
(\%)\end{array}$ & $\begin{array}{l}\text { FF } \\
(\%)\end{array}$ & $\begin{array}{l}\mathrm{V}_{\mathrm{OC}} \\
(\mathrm{V})\end{array}$ & $\begin{array}{c}\mathbf{J}_{\mathrm{sc}} \\
\left(\mathbf{m A} \cdot \mathbf{c m}^{-2}\right)\end{array}$ & Ref. \\
\hline H1 $\mathrm{MoSe}_{2} / \mathrm{WS}_{2}$ hybrid & 9.92 & 65.1 & 0.66 & 23.1 & $\begin{array}{l}\text { In this } \\
\text { work }\end{array}$ \\
\hline $\mathrm{CoSe}_{2} / \mathrm{C}$ on carbon cloth & 9.87 & 71 & 0.76 & 18.16 & 2 \\
\hline Multilayer $\mathrm{MoSe}_{2} / \mathrm{Mo}$ & 9.57 & 67 & 0.711 & 20.1 & 3 \\
\hline MOF-525/s-PT & 8.91 & 70 & 0.80 & 16.14 & 4 \\
\hline Nitrogen doped carbon nanowires & 8.90 & 74 & 0.75 & 16.08 & 5 \\
\hline $\mathrm{TiS}_{2}$-graphene & 8.8 & 68.5 & 0.72 & 17.76 & 6 \\
\hline Nitrogen-doped porous carbon & 8.75 & 69 & 0.77 & 16.54 & 7 \\
\hline $\begin{array}{l}\text { Magnetic carbon sphere }\left(\mathrm{CS} @ \mathrm{Fe}_{3} \mathrm{O}_{4}\right) \\
\text { on conductive glass }\end{array}$ & 8.74 & 71 & 0.859 & 14.33 & 8 \\
\hline $\mathrm{Fe}_{1-x} \mathrm{~S} / \mathrm{Fe}_{3} \mathrm{C}-\mathrm{NCNTs}-5$ & 8.67 & 67 & 0.80 & 16.19 & 9 \\
\hline $\mathrm{MoS}_{2} /$ carbon nanofibers & $8.46 \pm 0.08$ & 70.20 & 0.771 & 15.64 & 10 \\
\hline $\mathrm{WSe}_{2} / \mathrm{MoS}_{2}$ & 8.44 & 72.4 & 0.69 & 16.89 & 11 \\
\hline NiPt nanowires & 8.29 & 66.2 & 0.741 & 16.93 & 12 \\
\hline $\mathrm{CuS} / \mathrm{WS}_{2}$ & 8.21 & 72.16 & 0.72 & 15.77 & 13 \\
\hline In situ $\mathrm{MoSe}_{2} / \mathrm{Mo}$ & 8.13 & 67 & 0.805 & 15.07 & 14 \\
\hline B,N co-doped graphene & 8.08 & 69 & 0.77 & 15.3 & 15 \\
\hline $\mathrm{MoS}_{2}$ on GNTs & 8.01 & 60.01 & 0.74 & 18.14 & 16 \\
\hline $\mathrm{WS}_{2} / \mathrm{MoTe}_{2}$ & 7.99 & 69.69 & 0.74 & 15.34 & 17 \\
\hline $\begin{array}{l}\text { Nitrogen-doped core-shell carbon } \\
\text { spheres }\end{array}$ & 7.89 & 69.21 & 0.745 & 15.3 & 18 \\
\hline $\mathrm{MoS}_{2} / \mathrm{CNTs}$ & 7.83 & 66 & 0.74 & 16.65 & 19 \\
\hline $\mathrm{MoS}_{2} / \mathrm{S}$-doped carbon sphere & 7.72 & 68.34 & 0.725 & 15.58 & 20 \\
\hline $\mathrm{Co}_{3} \mathrm{O}_{4}-\mathrm{WC}-\mathrm{CN} / \mathrm{rGO}$ & 7.38 & 59 & 0.77 & 16.27 & 21 \\
\hline $\mathrm{WS}_{2} / \mathrm{MWCNT}$ & 7.36 & 72 & 0.75 & 13.63 & 22 \\
\hline $\mathrm{In}_{2.77} \mathrm{~S}_{4}$ nanoflakes/graphene & 7.32 & 66 & 0.74 & 14.92 & 23 \\
\hline Chemical bath $\mathrm{MoSe}_{2}$ & 7.28 & 73 & 0.699 & 14.33 & 24 \\
\hline Sputtered $\mathrm{MoTe}_{2}$ & 7.25 & 65.64 & 0.69 & 16.00 & 25 \\
\hline Ruthenium dioxide & 7.22 & 54 & 0.813 & 16.51 & 26 \\
\hline $\begin{array}{l}\text { Hierarchical architecture of } \\
\mathrm{MoSe}_{2} \text { nanoflowers }\end{array}$ & 7.01 & 57 & 0.695 & 17.85 & 27 \\
\hline $\mathrm{VO}_{2}(\mathrm{M}) / \mathrm{C}$ & 6.53 & 62.86 & 0.71 & 14.61 & 28 \\
\hline MWCNT@MoS & 6.45 & 65 & 0.73 & 13.69 & 29 \\
\hline
\end{tabular}




\begin{tabular}{|l|c|c|c|c|c|}
\hline Polyaniline - $\mathrm{MnO}_{2}$ on rGO & 6.15 & 65 & 0.74 & 12.88 & 30 \\
\hline $\begin{array}{l}\text { Composites of carbon black (86\%) and } \\
\text { 3D network polymers on stainless steel }\end{array}$ & 6.1 & 60.6 & 0.68 & 14.9 & 31 \\
\hline MWCNT/PEDOT:PSS & 5.03 & 62 & 0.72 & 11.27 & 32 \\
\hline
\end{tabular}


Table S4. The characteristic HER parameters of various electrocatalysts

\begin{tabular}{|c|c|c|c|}
\hline Electrode & $\begin{array}{c}\eta_{10} \\
(m V v s R H E)\end{array}$ & $\begin{array}{c}\text { Tafel } \\
\left(m V \cdot \text { dec }^{-1}\right)\end{array}$ & $\begin{array}{c}j_{0} \\
\left(m A \cdot c m^{-2}\right)\end{array}$ \\
\hline $\mathrm{MoSe}_{2}$ & 112 & 136 & 0.77 \\
\hline$W S_{2}$ & 158 & 114 & 0.41 \\
\hline$H 1$ & 75 & 60 & 1.19 \\
\hline$H 2$ & 78 & 64 & 2.29 \\
\hline$H 3$ & 83 & 70 & 2.16 \\
\hline$P t / C$ & 42 & 35 & 1.47 \\
\hline
\end{tabular}


Table S5. Electrocatalytic HER performances of TMD electrodes

\begin{tabular}{|c|c|c|c|c|c|}
\hline Electrocatalyst & $\begin{array}{c}\boldsymbol{\eta}(\mathbf{m V}) \\
\text { (a) } 10 \mathrm{~mA} / \mathrm{cm}^{2}\end{array}$ & $\begin{array}{l}\text { Tafel Slope } \\
\left(\mathrm{mV} \cdot \mathrm{dec}^{-1}\right)\end{array}$ & $\begin{array}{c}\mathbf{j}_{0} \\
\left(\mathbf{m A} \cdot \mathbf{c m}^{-2}\right)\end{array}$ & $\begin{array}{c}\text { Cdl } \\
\left(\mathrm{mF} \cdot \mathrm{cm}^{-2}\right)\end{array}$ & Ref \\
\hline H1 $\mathrm{MoSe}_{2} / \mathrm{WS}_{2}$ hybrid & 75 & 60 & 1.19 & 14.6 & This work \\
\hline Vertically aligned $\mathrm{MoSe}_{2}$ & 88 & 59.8 & 0.845 & - & 33 \\
\hline $\mathrm{MoSe}_{2}-\mathrm{Ni}_{3} \mathrm{Se}_{4}$ hybrid & 203 & 57 & - & 2.67 & 34 \\
\hline $\mathrm{WS}_{(1-x)} \mathrm{Se}_{x} /$ graphene/NF & 93 & 51 & 0.274 & - & 35 \\
\hline $\mathrm{Cu}$ doped $\mathrm{MoSe}_{2}$ & 86 & 44 & 0.212 & 2.27 & 36 \\
\hline $\mathrm{MoS}_{2} / \mathrm{WS}_{2}$ & 129 & 72 & 0.436 & - & 37 \\
\hline $\mathrm{SWCNTs} / \mathrm{MoSe}_{2}$ & 100 & 63 & $0.203 \times 10^{-3}$ & - & 38 \\
\hline $\mathrm{MoSe}_{2} / \mathrm{Bi}_{2} \mathrm{Se}_{3}$ hybrids & 300 & 44 & - & - & 39 \\
\hline $\mathrm{CoS}_{2} / \mathrm{CoSe}_{2} @ \mathrm{C}$ & 164 & 42 & - & 12.4 & 40 \\
\hline $1 \mathrm{~T}-\mathrm{WS}_{2}$ & 118 & 43 & - & - & 41 \\
\hline $\mathrm{MoSe}_{2}-\mathrm{NiSe} @$ carbon & 67 & 76.3 & 0.472 & 42.1 & 42 \\
\hline MoSex & 130 & 46 & - & 35.63 & 43 \\
\hline $\mathrm{MoSe}_{2} / \mathrm{CoP}$ & 105 & 51 & - & 20 & 44 \\
\hline H-TaS $2: H-\mathrm{TaSe}_{2}$ & 120 & - & - & 0.5 & 45 \\
\hline N-doped $\mathrm{MoSe}_{2}$ & 135 & 62 & - & - & 46 \\
\hline $\mathrm{MoS}_{2}-\mathrm{MoSe}_{2}$ & 175 & 46 & - & 37.78 & 47 \\
\hline Fe doped $\mathrm{MoSe}_{2}$ & 88 & 59 & 0.411 & 1.85 & 36 \\
\hline Pristine $\mathrm{MoSe}_{2}$ & 175 & 91 & - & - & 46 \\
\hline Zn doped $\mathrm{MoSe}_{2}$ & 182 & 58 & - & 28.3 & 48 \\
\hline $\mathrm{MoSe}_{2} @ \mathrm{MoS}_{2}$ & 161 & 60 & - & - & 49 \\
\hline $\mathrm{CoSe}_{2} / \mathrm{MoSe}_{2}$ & 218 & 76 & - & 4.3 & 50 \\
\hline Mn doped $\mathrm{MoSe}_{2}$ nanoflowers & 167 & 60 & - & 7 & 51 \\
\hline $\mathrm{MoS}_{2(1-\mathrm{X})} \mathrm{Se}_{2 \mathrm{x}}$ & 141 & 67 & 0.112 & - & $\sigma$ \\
\hline $\mathrm{WS}_{2(1-\mathrm{X})} \mathrm{Se}_{2 \mathrm{x}}$ & 167 & 107 & 0.0891 & - & \\
\hline Ni doped $\mathrm{MoSe}_{2}$ (NMS-15) & 180 & 67 & 4.0 & 5.84 & 53 \\
\hline
\end{tabular}




\begin{tabular}{|l|c|c|c|c|c|}
\hline Co doped $\mathrm{MoSe}_{2}$ (CMS-15) & 206 & 72 & 2.9 & 7.76 & \\
\hline $\mathrm{MoSe}_{2} /$ graphene network & 159 & 61 & - & - & 54 \\
\hline $\begin{array}{l}\mathrm{MoSe}_{2} \text { Nanosheets decorated } \\
\text { on carbon Fiber }\end{array}$ & 182 & 69 & 0.0233 & - & 55 \\
\hline $\mathrm{MoS}_{2} / \mathrm{WSe}_{2}$ & 116 & 76 & 0.0407 & 3.82 & 56 \\
\hline $\mathrm{CoSe}_{2}-\mathrm{WS}_{2}$ & 95 & 44 & 0.0144 & 3.2 & 57 \\
\hline $\mathrm{MoSe}_{2} /$ graphene/NF & 92 & 42 & 0.997 & 2.13 & 58 \\
\hline $\mathrm{MoSe}_{2} / \mathrm{rGO}$ & 186 & 61 & - & - & 59 \\
\hline
\end{tabular}

\section{References}

1. Zhang, Y.; Gao, L.; Hensen, E. J. M.; Hofmann, J. P., Evaluating the Stability of $\mathrm{Co}_{2} \mathrm{P}$ Electrocatalysts in the Hydrogen Evolution Reaction for Both Acidic and Alkaline Electrolytes. ACS Energy Lett. 2018, 3 (6), 1360-1365.

2. Chiu, I. T.; Li, C.-T.; Lee, C.-P.; Chen, P.-Y.; Tseng, Y.-H.; Vittal, R.; Ho, K.-C., Nanoclimbing-wall-like $\mathrm{CoSe}_{2} /$ Carbon Composite Film for the Counter Electrode of a Highly Efficient Dye-Sensitized Solar Cell: A Study on the Morphology Control. Nano Energy 2016, 22, 594-606.

3. Hussain, S.; Patil, S. A.; Vikraman, D.; Liu, H.; Kim, H.-S.; Jung, J., High Performance $\mathrm{MoSe}_{2} / \mathrm{Mo}$ Counter Electrodes Based- Dye-Sensitized Solar Cells. J. Electrochem. Soc. 2017, 164 (2), E11-E16.

4. Chen, T.-Y.; Huang, Y.-J.; Li, C.-T.; Kung, C.-W.; Vittal, R.; Ho, K.-C., Metal-Organic Framework/Sulfonated Polythiophene on Carbon Cloth as a Flexible Counter Electrode for Dye-Sensitized Solar Cells. Nano Energy 2017, 32, 19-27.

5. Meng, X.; Yu, C.; Zhang, X.; Huang, L.; Rager, M.; Hong, J.; Qiu, J.; Lin, Z., Active Sites-Enriched Carbon Matrix Enables Efficient Triiodide Reduction in DyeSensitized Solar Cells: An Understanding of the Active Centers. Nano Energy 2018, 54, 138147.

6. Meng, X.; Yu, C.; Lu, B.; Yang, J.; Qiu, J., Dual Integration System Endowing Two-Dimensional Titanium Disulfide with Enhanced Triiodide Reduction Performance in Dye-Sensitized Solar Cells. Nano Energy 2016, 22, 59-69.

7. Xiao, N.; Song, J.; Wang, Y.; Liu, C.; Zhou, Y.; Liu, Z.; Li, M.; Qiu, J., Nitrogen-doped Porous Carbon with Well-Balanced Charge Conduction and Electrocatalytic Activity for Dye-Sensitized Solar Cells. Carbon 2018, 128, 201-204. 
8. Gao, C.; Wang, H.; Han, Q.; Hu, Z.; Wu, M., High-Efficiency Magnetic Carbon Spheres Counter Electrode for Dye-Sensitized Solar Cell. Electrochim. Acta 2018, 264, 312318.

9. $\quad$ Chen, M.; Wang, G.-C.; Shao, L.-L.; Yuan, Z.-Y.; Qian, X.; Jing, Q.-S.; Huang, Z.-Y.; Xu, D.-L.; Yang, S.-X., Strategic Design of Vacancy-Enriched $\mathrm{Fe}_{1-\mathrm{X}} \mathrm{S}$ Nanoparticles Anchored on $\mathrm{Fe}_{3} \mathrm{C}$-Encapsulated and N-Doped Carbon Nanotube Hybrids for High-Efficiency Triiodide Reduction in Dye-Sensitized Solar Cells. ACS Appl. Mater. Interfaces 2018, 10 (37), 31208-31224.

10. Li, L.; Zhang, X.; Wang, D.; Zhang, W.; Li, X.; Zhao, X.; Zhang, Q.;

Gu, L.; Yu, Z.; Wu, M., Electrospinning Synthesis of High Performance Carbon Nanofiber Coated Flower-like $\mathrm{MoS}_{2}$ Nanosheets for Dye-Sensitized Solar Cells Counter Electrode. Electrochim. Acta 2018, 280, 94-100.

11. Vikraman, D.; Arbab, A. A.; Hussain, S.; Shrestha, N. K.; Jeong, S. H.; Jung, J.; Patil, S. A.; Kim, H.-S., Design of $\mathrm{WSe}_{2} / \mathrm{MoS}_{2}$ Heterostructures as the Counter Electrode to Replace Pt for Dye-Sensitized Solar Cell. ACS Sustain. Chem. Eng. 2019, 7 (15), 1319513205.

12. Pang, Z.; Zhao, Y.; Duan, Y.; Duan, J.; Tang, Q.; Yu, L., Well-aligned NiPt Alloy Counter Electrodes for High-Efficiency Dye-Sensitized Solar Cell Applications. J. Energy Chem. 2019, 30, 49-56.

13. Hussain, S.; Patil, S. A.; Memon, A. A.; Vikraman, D.; Naqvi, B. A.; Jeong, S. H.; Kim, H.-S.; Kim, H.-S.; Jung, J., CuS/WS ${ }_{2}$ and $\mathrm{CuS} / \mathrm{MoS}_{2}$ Heterostructures for High Performance Counter Electrodes in Dye-Sensitized Solar Cells. Solar Energy 2018, 171, 122129.

14. Chen, H.; Xie, Y.; Cui, H.; Zhao, W.; Zhu, X.; Wang, Y.; Lü, X.; Huang, F., In situ Growth of a MoSe 2 /Mo Counter Electrode for High Efficiency Dye-Sensitized Solar Cells. Chem. Commun. 2014, 50 (34), 4475-4477.

15. Yu, C.; Fang, H.; Liu, Z.; Hu, H.; Meng, X.; Qiu, J., Chemically Grafting Graphene Oxide to B,N co-doped Graphene via Ionic Liquid and Their Superior Performance for Triiodide Reduction. Nano Energy 2016, 25, 184-192.

16. Yu, F.; Shi, Y.; Shen, X.; Yao, W.; Han, S.; Ma, J., Three-Dimensional $\mathrm{MoS}_{2^{-}}$ Nanosheet-Based Graphene/Carbon Nanotube Aerogel as a Pt-Free Counter Electrode for High-Efficiency Dye-Sensitized Solar Cells. ACS Sustain. Chem. Eng. 2018, 6 (12), $17427-$ 17434. 
17. Hussain, S.; Patil, S. A.; Memon, A. A.; Vikraman, D.; Abbas, H. G.; Jeong, S. H.; Kim, H.-S.; Kim, H.-S.; Jung, J., Development of a $\mathrm{WS}_{2} / \mathrm{MoTe}_{2}$ Heterostructure as a Counter Electrode for the Improved Performance in Dye-Sensitized Solar Cells. Inorg. Chem. Front. 2018, 5 (12), 3178-3183.

18. Wang, W.; Yao, J.; Zuo, X.; Li, G., High Efficiency Nitrogen-Doped Core-Shell Carbon Spheres as Counter Electrodes for Dye-Sensitized Solar Cells. Mater. Lett. 2018, 227, $172-175$.

19. Lin, C.-H.; Tsai, C.-H.; Tseng, F.-G.; Ma, C.-C. M.; Wu, H.-C.; Hsieh, C.-K., Three-dimensional Vertically Aligned Hybrid Nanoarchitecture of Two-dimensional Molybdenum Disulfide Nanosheets Anchored on Directly Grown One-dimensional Carbon Nanotubes for Use as a Counter Electrode in Dye-Sensitized Solar Cells. J. Alloys Compd. 2017, 692, 941-949.

20. Ding, Y.; Huang, N.; Yan, S.; Peng, R.; Sun, P.; Sun, X.; Huang, C., A $\mathrm{MoS}_{2} /$ Sulfur-doped Carbon Sphere Nanohybrid Catalyst with High-Efficiency Electrocatalysis for Flexible Counter Electrodes. J. Alloys Compd. 2018, 767, 848-855.

21. Chen, L.; Chen, W.; Wang, E., Graphene with Cobalt Oxide and Tungsten Carbide as a Low-cost Counter Electrode Catalyst Applied in Pt-free Dye-Sensitized Solar Cells. $J$. Power Sources 2018, 380, 18-25.

22. Wu, J.; Yue, G.; Xiao, Y.; Huang, M.; Lin, J.; Fan, L.; Lan, Z.; Lin, J.-Y., Glucose Aided Preparation of Tungsten Sulfide/Multi-Wall Carbon Nanotube Hybrid and Use as Counter Electrode in Dye-Sensitized Solar Cells. ACS Appl. Mater. Interfaces 2012, 4 (12), 6530-6536.

23. Zhou, B.; Zhang, X.; Jin, P.; Li, X.; Yuan, X.; Wang, J.; Liu, L., Synthesis of $\mathrm{In}_{2.77} \mathrm{~S}_{4}$ Nanoflakes/Graphene Composites and Their Application as Counter Electrode in DyeSensitized Solar Cells. Electrochim. Acta 2018, 281, 746-752.

24. Vikraman, D.; Patil, S. A.; Hussain, S.; Mengal, N.; Jeong, S. H.; Jung, J.; Park, H. J.; Kim, H. S.; Kim, H. S., Construction of Dye-Sensitized Solar Cells Using Wet Chemical Route Synthesized MoSe ${ }_{2}$ Counter Electrode. J. Ind. Eng. Chem. 2019, 69, 379-386. 25. Hussain, S.; Patil, S. A.; Vikraman, D.; Mengal, N.; Liu, H.; Song, W.; An, K.-S.; Jeong, S. H.; Kim, H.-S.; Jung, J., Large area growth of $\mathrm{MoTe}_{2}$ films as high performance counter electrodes for dye-sensitized solar cells. Sci. Rep. 2018, 8 (1), 29. 
26. Hou, Y.; Chen, Z. P.; Wang, D.; Zhang, B.; Yang, S.; Wang, H. F.; Hu, P.; Zhao, H. J.; Yang, H. G., Highly Electrocatalytic Activity of $\mathrm{RuO}_{2}$ Nanocrystals for Triiodide Reduction in Dye-Sensitized Solar Cells. Small 2014, 10 (3), 484-492.

27. Yuan, X.; Zhou, B.; Zhang, X.; Li, Y.; Liu, L., Hierarchical MoSe $\mathrm{M}_{2}$ Nanoflowers Used as Highly Efficient Electrode for Dye-Sensitized Solar Cells. Electrochim. Acta 2018, 283, 1163-1169.

28. Gnanasekar, S.; Kollu, P.; Jeong, S. K.; Grace, A. N., Pt-Free, Low-Cost and Efficient Counter Electrode With Carbon Wrapped $\mathrm{VO}_{2}(\mathrm{M})$ Nnanofiber for Dye-Sensitized Solar Cells. Sci. Rep. 2019, 9 (1), 5177.

29. Tai, S.-Y.; Liu, C.-J.; Chou, S.-W.; Chien, F. S.-S.; Lin, J.-Y.; Lin, T.-W., Few-layer $\mathrm{MoS}_{2}$ Nanosheets Coated onto Multi-walled Carbon Nanotubes as a Low-cost and Highly Electrocatalytic Counter Electrode for Dye-Sensitized Solar Cells. J. Mater. Chem. 2012, 22 (47), 24753-24759.

30. Sun, W.; Peng, T.; Liu, Y.; Xu, S.; Yuan, J.; Guo, S.; Zhao, X.-Z., Hierarchically Porous Hybrids of Polyaniline Nanoparticles Anchored on Reduced Graphene Oxide Sheets as Counter Electrodes for Dye-Sensitized Solar Cells. J. Mater. Chem. A 2013, 1 (8), 2762-2768.

31. Kang, G.; Choi, J.; Park, T., Pt-Free Counter Electrodes with Carbon Black and 3D Network Epoxy Polymer Composites. Sci. Rep. 2016, 6, 22987.

32. Abid, A.; Syed Mujtaba, S.; Sinem, B.; Mehmet, K.; Bahadır, K.; $\quad$ Murat, K.; Salih, A.; Serap, G., Metal-free Polymer/MWCNT Composite Fiber as an Efficient Counter Electrode in Fiber Shape Dye-Sensitized Solar Cells. Nanotechnology 2016, 27 (38), 384003. 33. Vikraman, D.; Hussain, S.; Akbar, K.; Adaikalam, K.; Lee, S. H.; Chun, S. H.; Jung, J.; Kim, H. S.; Park, H. J., Facile Synthesis of Molybdenum Diselenide Layers for High-Performance Hydrogen Evolution Electrocatalysts. ACS Omega 2018, 3 (5), 57995807.

34. Wu, P.; Sun, G.; Chen, Y.; Xu, W.; Zheng, H.; Xu, J.; Wang, L.; Peng, D.L., $\mathrm{MoSe}_{2}-\mathrm{Ni}_{3} \mathrm{Se}_{4}$ Hybrid Nanoelectrocatalysts and Their Enhanced Electrocatalytic Activity for Hydrogen Evolution Reaction. Nanoscale Res. Lett. 2020, 15 (1), 132.

35. Hussain, S.; Akbar, K.; Vikraman, D.; Afzal, A. R.; Song, W.; An, K.-S.; Farooq, A.; Park, J.-Y.; Chun, S.-H.; Jung, J., WS $\mathrm{W}_{(1-\mathrm{x})} \mathrm{Se}_{\mathrm{x}}$ Nanoparticles Decorated ThreeDimensional Graphene on Nickel Foam: A Robust and Highly Efficient Electrocatalyst for the Hydrogen Evolution Reaction. Nanomaterials 2018, 8 (11), 929. 
36. Vikraman, D.; Hussain, S.; Akbar, K.; Karuppasamy, K.; Chun, S. H.; Jung, J.; Kim, H. S., Design of Basal Plane Edges in Metal-Doped Nanostripes-Structured $\mathrm{MoSe}_{2}$ Atomic Layers to Enhance Hydrogen Evolution Reaction Activity. ACS Sustain. Chem. Eng. 2019, 7 (1), 458-469.

37. Vikraman, D.; Hussain, S.; Akbar, K.; Truong, L.; Kathalingam, A.; Chun, S.-H.; Jung, J.; Park, H. J.; Kim, H.-S., Improved Hydrogen Evolution Reaction Performance using $\mathrm{MoS}_{2}-\mathrm{WS}_{2}$ Heterostructures by Physicochemical Process. ACS Sustain. Chem. Eng. 2018, 6 (7), 8400-8409.

38. Leyla, N.; Sebastiano, B.; Reinier, O.-N.; Alberto, A.; Mirko, P.; Esau, D. R. C. A.; Francesco, B., Engineered $\mathrm{MoSe}_{2}$-Based Heterostructures for Efficient Electrochemical Hydrogen Evolution Reaction. Adv. Energy Mater. 2018, 8 (16), 1703212.

39. Yang, J.; Wang, C.; Ju, H.; Sun, Y.; Xing, S.; Zhu, J.; Yang, Q., Integrated Quasiplane Heteronanostructures of $\mathrm{MoSe}_{2} / \mathrm{Bi}_{2} \mathrm{Se}_{3}$ Hexagonal Nanosheets: Synergetic Electrocatalytic Water Splitting and Enhanced Supercapacitor Performance. Adv. Funct. Mater. 2017, 27 (48), 1703864.

40. Karuppasamy, K.; Bose, R.; Jothi, V. R.; Vikraman, D.; Jeong, Y.-T.; Arunkumar, P.; Velusamy, D. B.; Maiyalagan, T.; Alfantazi, A.; Kim, H.-S., High Performance, 3D-Hierarchical $\mathrm{CoS}_{2} / \mathrm{CoSe} @ \mathrm{C}$ Nanohybrid as an Efficient Electrocatalyst for Hydrogen Evolution Reaction. J. Alloys Compd. 2020, 838, 155537.

41. He, Q.; Wang, L.; Yin, K.; Luo, S., Vertically Aligned Ultrathin $1 \mathrm{~T}-\mathrm{WS}_{2}$ Nanosheets Enhanced the Electrocatalytic Hydrogen Evolution. Nanoscale Res. Lett. 2018, 13 (1), 167.

42. Liu, C.; Wang, K.; Zheng, X.; Liu, X.; Liang, Q.; Chen, Z., Rational Design of $\mathrm{MoSe}_{2}$-NiSe@Carbon Heteronanostructures for Efficient Electrocatalytic Hydrogen Evolution in Both Acidic and Alkaline Media. Carbon 2018, 139, 1-9.

43. Kwon, I. S.; Kwak, I. H.; Debela, T. T.; Abbas, H. G.; Park, Y. C.; Ahn, J.p.; Park, J.; Kang, H. S., Se-Rich $\mathrm{MoSe}_{2}$ Nanosheets and Their Superior Electrocatalytic Performance for Hydrogen Evolution Reaction. ACS Nano 2020, 14 (5), 6295-6304.

44. Zhang, L.; Zhu, J.; Wang, Z.; Zhang, W., 2D MoSe 2 /CoP Intercalated Nanosheets for Efficient Electrocatalytic Hydrogen Production. Int. J. Hydrog. Energy 2020, 45 (38), 19246-19256.

45. Najafi, L.; Bellani, S.; Oropesa-Nuñez, R.; Martín-García, B.; Prato, M.; Pasquale, L.; Panda, J.-K.; Marvan, P.; Sofer, Z.; Bonaccorso, F., TaS 2 , TaSe 2 , and Their 
Heterogeneous Films as Catalysts for the Hydrogen Evolution Reaction. ACS Catal. 2020, 10 (5), 3313-3325.

46. Ren, X.; Ma, Q.; Ren, P.; Wang, Y., Synthesis of Nitrogen-doped $\mathrm{MoSe}_{2}$ Nanosheets with Enhanced Electrocatalytic Activity for Hydrogen Evolution Reaction. Int. J. Hydrog. Energy 2018, 43 (32), 15275-15280.

47. Yang, J.; Zhu, J.; Xu, J.; Zhang, C.; Liu, T., MoSe ${ }_{2}$ Nanosheet Array with Layered $\mathrm{MoS}_{2}$ Heterostructures for Superior Hydrogen Evolution and Lithium Storage Performance. ACS Appl. Mater. Interfaces 2017, 9 (51), 44550-44559.

48. Qian, J.; Wang, T.; Xia, B.; Xi, P.; Gao, D., Zn-doped $\mathrm{MoSe}_{2}$ Nanosheets as High-Performance Electrocatalysts for Hydrogen Evolution Reaction in Acid Media. Electrochim. Acta 2019, 296, 701-708.

49. Ren, X.; Wei, Q.; Ren, P.; Wang, Y.; Chen, R., Synthesis of Flower-like $\mathrm{MoSe}_{2} @ \mathrm{MoS}_{2}$ Nanocomposites as the High Efficient Water Splitting Electrocatalyst. Mater. Lett. 2018, 231, 213-216.

50. Zhao, G.; Li, P.; Rui, K.; Chen, Y.; Dou, S. X.; Sun, W., $\mathrm{CoSe}_{2} / \mathrm{MoSe}_{2}$ Heterostructures with Enriched Water Adsorption/Dissociation Sites towards Enhanced Alkaline Hydrogen Evolution Reaction. Chem.: $\quad$ Eur. J. 2018, 24 (43), 11158-11165.

51. Kuraganti, V.; Jain, A.; Bar-Ziv, R.; Ramasubramaniam, A.; Bar-Sadan, M., Manganese Doping of $\mathrm{MoSe}_{2}$ Promotes Active Defect Sites for Hydrogen Evolution. ACS Appl. Mater. Interfaces 2019, 11 (28), 25155-25162.

52. Hussain, S.; Akbar, K.; Vikraman, D.; Karuppasamy, K.; Kim, H. S.; Chun, S. H.; Jung, J., Synthesis of $\mathrm{MoS}_{2(1-X)} \mathrm{Se}_{2 \mathrm{x}}$ and $\mathrm{WS}_{2(1-\mathrm{X})} \mathrm{Se}_{2 \mathrm{x}}$ Alloys for Enhanced Hydrogen Evolution Reaction Performance. Inorg. Chem. Front. 2017, 4 (12), 2068-2074.

53. Zhao, G.; Wang, X.; Wang, S.; Rui, K.; Chen, Y.; Yu, H.; Ma, J.; Dou, S. X.; Sun, W., Heteroatom-doped $\mathrm{MoSe}_{2}$ Nanosheets with Enhanced Hydrogen Evolution Kinetics for Alkaline Water Splitting. Chem. Asian J. 2019, 14 (2), 301-306.

54. Mao, S.; Wen, Z.; Ci, S.; Guo, X.; Ostrikov, K.; Chen, J., Perpendicularly Oriented $\mathrm{MoSe}_{2} /$ Graphene Nanosheets as Advanced Electrocatalysts for Hydrogen Evolution. Small 2015, 11 (4), 414-419.

55. Qu, B.; Yu, X.; Chen, Y.; Zhu, C.; Li, C.; Yin, Z.; Zhang, X., Ultrathin $\mathrm{MoSe}_{2}$ Nanosheets Decorated on Carbon Fiber Cloth as Binder-Free and High-Performance Electrocatalyst for Hydrogen Evolution. ACS Appl. Mater. Interfaces 2015, 7 (26), 1417014175 . 
56. Vikraman, D.; Hussain, S.; Truong, L.; Karuppasamy, K.; Kim, H. J.; Maiyalagan, T.; Chun, S. H.; Jung, J.; Kim, H. S., Fabrication of $\mathrm{MoS}_{2} / \mathrm{WSe}_{2}$ Heterostructures as Electrocatalyst for Enhanced Hydrogen Evolution Reaction. Appl. Surf. Sci. 2019, 480, 611-620.

57. Hussain, S.; Akbar, K.; Vikraman, D.; Liu, H.; Chun, S. H.; Jung, J., $\mathrm{WS}_{2} / \mathrm{CoSe}_{2}$ Heterostructure: A Designed Structure as Catalysts for Enhanced Hydrogen Evolution Performance. J. Ind. Eng. Chem. 2018, 65, 167-174.

58. Hussain, S.; Vikraman, D.; Akbar, K.; Naqvi, B. A.; Abbas, S. M.; Kim, H.S.; Chun, S.-H.; Jung, J., Fabrication of $\mathrm{MoSe}_{2}$ Decorated Three-Dimensional Graphene Composites Structure as a Highly Stable Electrocatalyst for Improved Hydrogen Evolution Reaction. Renew. Energy 2019, 143, 1659-1669.

59. Sarker, S.; Peters, J.; Chen, X.; Li, B.; Chen, G.; Yan, L.; Richins, S. K.; Das, S.; Zhou, M.; Luo, H., Engineering Molybdenum Diselenide and Its Reduced Graphene Oxide Hybrids for Efficient Electrocatalytic Hydrogen Evolution. ACS Appl. Nano Mater. 2018, 1 (5), 2143-2152. 